Harold A. McAlister and William I. Harkopf (eds.)

\title{
Speckle Interferometry at the U. S. Naval Observatory
}

\author{
CHARLES E. WORLEY \& GEOFFREY G. DOUGLASS \\ U. S. Naval Observatory, Washington, DC, 20392-5100, USA
}

\begin{abstract}
A new speckle interferometer began operation with the 66-cm refractor in Oct. 1990. The interferometer is an improved version of the one operated by CHARA at Georgia State University. We describe the instrument, its calibration, and first observational results.
\end{abstract}

\section{INTRODUCTION}

Our long term interest in the astrometric observation of visual double stars, coupled with the evident success and precision of the speckle interferometer developed by McAlister et. al. $(1987, A J, \mathbf{9 3}, 688)$ at Georgia State University, led us to plan for a similar, but improved, instrument for the U. S. Naval Observatory, beginning in 1987 . This contribution describes the instrument, its initial calibration, and the first observational results attained with it.

Because we anticipated that this interferometer would be attached regularly to the $66-\mathrm{cm}$ refractor, with its nearly $5-\mathrm{m}$ moment arm, but that we also might wish to transport it to other sites, we specified a light, modular construction. Total weight of the detector, camera, control unit, and power supply is about $20 \mathrm{~kg}$. Camera control, data, and high-voltage lines are threaded through the telescope tube and mounting into adjacent office space which contains the camera control and data reduction computers, as well as video monitors, tape recorder, and voltage gain controller. Thus, an observing session requires two persons: one, in the dome, selecting stars and setting the telescope; the second, in the office, making the proper camera settings and voltage gain, and monitoring the on-line data reduction process. Communication is secured through an intercom system. We now proceed to a more detailed description of the speckle system components.

\section{CAMERA HEAD \& DETECTOR MODULE}

The camera head consists of four sub-assemblies; collimating lens, filter wheel, Risley prism, and focusing lens. The collimating lens assembly consists of two microscope objectives mounted on a motorized wheel, and an aberration correction lens to allow collimation of the light from an on-axis image. Various microscope objectives, ranging from 3.5 to $20 \mathrm{X}$, are available. A $10 \mathrm{X}$ objective is used routinely.

The filter wheel sub-assembly has space for six filters. Taking into account the focal curve of the refractor, we have found the following filters most useful: The $\mathrm{V}$ and $\mathrm{y}$ filters are standard, while the " $\mathrm{g}$ " filter is of our own concoction, consisting of $1 \mathrm{~mm} \mathrm{OG} 530$ and $3 \mathrm{~mm}$ BG 039. Of course, one has also to understand that the attainable magnitude is critically dependent on both seeing and transparency. 


\begin{tabular}{|cccc|}
\hline Filter & $\begin{array}{c}\lambda \\
(\AA)\end{array}$ & $\begin{array}{c}\text { Half-width } \\
(\AA)\end{array}$ & $\begin{array}{c}\text { Max. Mag. } \\
(\mathrm{V})\end{array}$ \\
\hline $\mathrm{V}$ & $\mathbf{5 4 5 0}$ & $\mathbf{8 5 0}$ & $9.5-10:$ \\
“g” & $\mathbf{5 4 0 0}$ & 610 & $9.0-9.5$ \\
$\mathrm{y}$ & $\mathbf{5 4 7 0}$ & 230 & $\mathbf{8 . 2}-\mathbf{8 . 5}$ \\
\hline
\end{tabular}

The Risley prism assembly consists of two prisms in a rotatable assembly, coupled to absolute encoders to monitor position for atmospheric dispersion compensation. The encoders have 360 positions per revolution, and are independently controlled by commands from the Instrument Computer.

The focus lens sub-assembly consists of an achromatic doublet so mounted that axial adjustment of the lens is allowed to change the focus position of the re-imaged object on to the detector.

Camera head control electronics are housed in a box attached to the camera head. Central to this unit is a single-board computer used to control the optical sub-assemblies in the camera head. This computer receives high level commands from the Instrument Computer, then reads and sets the positions of encoders and the relay board to control motors which in turn properly set the microscopic objectives, filters, and Risley prisms.

The detector module consists of an ICCD camera, its high-voltage controller, and associated light shield and mounting bracket. The ICCD camera, produced by ITT, uses an $18 \mathrm{~mm}$ single microchannel plate image intensifier fiberoptically coupled to a TI CCD. The camera produces shot noise limited performance. Gating may be manually adjusted over a range of 1 to $33 \mathrm{msec}$, and output video from the camera is in RS-170 format.

\section{INSTRUMENT \& REDUCTION COMPUTERS}

Both computers have 80386 processors operating at $25 \mathrm{Mhz}$, and identical hardware, including 110 Mbyte hard disks, floppy drives, VGA monitors, streaming tape drives, printers, Imaging Technology video digitizers, and operate under the MS DOS 4.01 system. They thus can be interchanged, if necessary. However, their software reflects their tasks.

Custom designed software for the instrument(observing) computer includes a camera control menu, observing list and $\log$, real time DVA (directed vector autocorrelation) and VA (vector autocorrelation), interface to VGA graphics display, quick look image processing, data storage, and an archived data base. Custom software for the reduction computer includes DVA and VA processing software, background and centroiding algorithms, and observing program and archived data bases.

The video output of the detector is archived on $8 \mathrm{~mm}$ tape, and the digitized data is stored (temporarily) on hard disk. Periodically, the disk data is archived by use of 150 Mbyte streaming tapes. The operator monitors real-time image appearence on a video screen. Another screen enables him to observe the autocorrelogram as the reductions proceed. At a later time, the data is recovered from disk and fit with paraboloids, and position angle and separation derived. 


\section{CALIBRATION}

There are many examples in the literature of speckle measures where poor calibration evidently has impaired the quality of the results. For this kind of small field astrometry, we are concerned primarily with the classical problems of orientation and scale, as well as with an artifact of the video system called the pitch, which is the ratio of $x$ to $y$.

Hitherto, we have operated with approximate values of the three quantities derived from binaries of known position angle and separation. We now plan to determine independent values of these constants in several ways. For orientation, we are taking trails under good seeing conditions. These have been fitted and reduced for us by the Georgia State group. Upon its emergence from near eclipse, we also plan to observe the edge-on system $\alpha$ Com. For scale and pitch, we have experimented with one of the objective gratings originally constructed for the photographic double star program. While such gratings traditionally have been used for magnitude compensation, there is no reason why a precision grating can not yield good determinations of scale and pitch. We plan to use such a grating "crossed"; that is, vertical and then horizontal, which is easily possible with the present grating rotation mechanism on our refractor.

\section{CURRENT PROGRAM \& RESULTS}

Our program with the $66-\mathrm{cm}$ refractor has two aims: first, to provide quality speckle measurements of all the pairs within range of our equipment, and second, thereby to provide a bridge between the classical astrometry of double stars and the new speckle technique.

At present, we have made more than 1800 measures of approximately 425 pairs. It is our intent to make repeated measures of each pair in the same season, as well as on a continuing basis for moving pairs and certain "standards". This appears to be an attainable and realistic goal.

At present, for bright pairs of small magnitude difference, where we can use the Strömgren y filter, we attain an internal standard deviation of 0.01 $0^{\prime \prime} .02$. This grows, of course, for fainter pairs and those with larger magnitude differences, where the wider band filters must be used. But a definitive study of our errors awaits better determinations of the astrometric and instrumental constants.

\section{ACKNOWLEDGEMENTS}

We are immeasurably indebted to H. McAlister, W. Hartkopf, D. Barry, and their many colleagues at Georgia State University for advice and support, as well as for the extensive software supplied by them. We wish to compliment W. Robinson of Systems Engineering Associates, for producing an instrument which worked the first time it was attached, and which has continued to work reliably. We also wish to thank G. Westerhout and the late J. Hughes for their support throughout the project. 